\title{
Registration for deceased organ and tissue donation among Ontario immigrants: a population-based cross-sectional study
}

\author{
Alvin Ho-ting Li BHSc, Ngan N. Lam MD MSc, Sonny Dhanani MD, Matthew Weir MD MSc, \\ Versha Prakash MHA, Joseph Kim MD PhD, Greg Knoll MD MSc, Amit X. Garg MD PhD
}

\section{Abstract}

Background: Canada has low rates of deceased organ and tissue donation. Immigrants to Canada may differ in their registered support for deceased organ donation based on their country of origin.

Methods: We used linked administrative databases in Ontario (about 11 million residents aged $\geq 16$ yr) to study the proportion of immigrants and long-term residents registered for deceased organ and tissue donation as of October 2013. We used modified Poisson regression to identify and quantify predictors of donor registration.

Results: Compared with long-term residents $(n=9244570)$, immigrants $(n=1947646)$ were much less likely to register for deceased organ and tissue donation (11.9\% v. $26.5 \%$ ). Immigrants from the United States, Australia and New Zealand had the highest registration rate $(40.0 \%)$, whereas immigrants with the lowest registration rates were from Eastern Europe and Central Asia $(9.4 \%)$, East Asia and Pacific $(8.4 \%)$ and sub-Saharan Africa $(7.9 \%)$. The largest numbers of unregistered immigrants were from India $(n=202548)$, China $(n=186678)$ and the Philippines $(n=125686)$. Characteristics among the immigrant population associated with a higher likelihood of registration included economic immigrant status, living in a rural area (population $<10000$ ), living in an area with a lower ethnic concentration, less material deprivation, a higher education, ability to speak English and French, and more years residing in Canada.

Interpretation: Immigrants in Ontario were less likely to register for deceased organ and tissue donation than long-term residents. There is a need to better understand reasons for lower registration rates among Canadian immigrants and to create culture-sensitive materials to build support for deceased organ and tissue donation.

\footnotetext{
T ncreasing rates of organ and tissue donation in Canada could help save the lives of the thousands of Canadians with end-stage organ failure. One factor that can influence the family's decision to consent to organ donation is having knowledge of the potential donor's wishes in this regard. ${ }^{1}$ Canada has an "opt-in" system, whereby citizens can record their donation wishes through a deceased organ donor registry, which can then be used to inform family members in the event of death., ${ }^{2,3}$ Increasing the number of registered donors is a key strategy adopted by Canadian organ procurement organizations to improve organ and tissue donation. ${ }^{2}$ An important step to increase the registration rate is to identify subpopulations that have lower donor registration rates and to better understand the reasons for nonregistration.
}

Ethnic minority populations have greater concerns regarding organ donation compared with the general population, and these concerns may be culture-specific. ${ }^{4}$ Documented issues include medical mistrust among the black population, ${ }^{4-6}$ religious uncertainties among North Americans of the Islamic faith, ${ }^{4-6}$ donor registry unawareness among Chinese and IndoAsian Canadians ${ }^{7,8}$ and lack of societal integration among

Competing interests: None declared.

This article has been peer reviewed.

Correspondence to: Alvin Li, Alvin.li@Ihsc.on.ca

CMAJ Open 2016. DOI:10.9778/cmajo.20160024 
Arab Americans. ${ }^{9}$ In addition, most new immigrants to Canada are from regions with less-developed organ donation systems that lack donor registries, such as Asia, Latin America and the Caribbean. ${ }^{10}$ As rates of migration continue to rise in Canada and other countries, identifying immigrant groups with lower donor registration rates and understanding how sociodemographic factors can affect organ and tissue donor registration can inform culturally sensitive donation practice, public education and awareness campaigns. ${ }^{4,11}$

To better inform these areas, we conducted a populationbased study in Ontario to determine the registration status for deceased organ and tissue donation for over 1 million recent immigrants from different countries compared with longterm residents. Our main outcome of interest was the proportion of immigrants and long-term residents of Canada who were registered deceased organ and tissue donors. For recent immigrants, we examined characteristics independently associated with registering for deceased organ and tissue donation. We also identified the 5 countries with the largest absolute numbers of unregistered people. Our secondary outcome was whether registered donors opted to exclude certain organs or tissues that they did not wish to donate.

\section{Methods}

\section{Design and setting}

We conducted a population-based cross-sectional study using linked health care databases in Ontario as of Oct. 22, 2013. These datasets were linked with the use of unique encoded identifiers and analyzed at the Institute for Clinical Evaluative Sciences. Ontario is Canada's most populous province (accounting for about $40 \%$ of the Canadian population), ${ }^{12}$ with about 11 million residents aged 16 years (the minimum age required to register for deceased organ and tissue donation) or more. In a 2012 study comparing characteristics and proportions registered of various registries worldwide, Nova Scotia had the highest proportion of citizens registered, at $65 \%$, followed by New Brunswick (42\%), Ontario (18\%), Yukon Territory (16\%), Quebec (10\%) and British Columbia $(8 \%) .^{2}$ In Canada, donor registries are "opt-in" registries that record only "yes" responses. People can register for organ and tissue donation when they renew or apply for a new driver's licence or health card at ServiceOntario. Registration can also be completed online. Those who choose to register can select the option to exclude certain organs or tissues from donation. To register as an organ and tissue donor in Ontario, one must be at least 16 years old and have a valid health card (https:// www.ontario.ca/page/organ-and-tissue-donor-registration).

\section{Data sources}

We ascertained sociodemographic and donor registration information from 2 main administrative databases: the Ontario Registered Persons Database and Immigration, Refugees and Citizenship Canada's Permanent Resident Database. The former contains demographic information and donor registration for all residents of Ontario who have ever been issued a health card by the Ontario government. This captures every- one who has registered for organ donation. We derived income using neighbourhood income quintiles (a measure of income adjusted to household size). ${ }^{13}$ Marginalization is the "process by which individuals and groups are prevented from fully participating in society." 14 We used Matheson and colleagues' ${ }^{15}$ Canadian Marginalization Index to assign marginalization quintiles based on area of residence on 4 components of marginalization: residential instability (a measure of turnover in the population), ethnic concentration (a measure consisting of the proportions of recent immigrants and of people who self-identify as a visible minority), dependency (a ratio measure of the dependent population [i.e., seniors and children] to the working-age population) and material deprivation (a measure of inability to afford consumption goods or services). This index was developed with the use of a theoretical framework, derived from census indicators and created by sorting the data into 5 quintiles (from least marginalized to most marginalized).

The Permanent Resident Database contains landing records for every permanent legal immigrant to Canada who arrived in 1985 or later. Data are captured at the time of immigration application. ${ }^{16} \mathrm{We}$ used the Ontario portion of this database to ascertain immigration status and other migration-related variables. The database has been used previously to examine diabetes and cancer screening among immigrants. ${ }^{17,18}$ The migration-related variables included time since arrival in Canada, immigration visa class (economic, family, refugee or other), language ability (English, French, both or neither), marital status (married, single, separated, divorced or widowed) and education level at the time of immigration. "Economic" immigrants included those sponsored by the province, skilled workers, entrepreneurs and investors. "Family" immigrants were family members of economic immigrants and those who immigrated through family reunification. The "other" category included all other immigrant classes, such as live-in caregivers and those who immigrated on humanitarian grounds.

\section{Study population}

We included all permanent residents of Ontario as of October 2013 and classified them as immigrants or long-term residents based on their immigration status within the Permanent Resident Database. Long-term residents were those who did not have a record in the database. We excluded people who did not make at least 1 contact with the health care system in the 5 years before October 2013 to ensure we included only people in Ontario.

\section{Outcomes}

We used each immigrant's country of birth to categorize most immigrants by world region of origin, according to the World Bank system: Western Europe; Eastern Europe and Central Asia; Middle East and North Africa; sub-Saharan Africa; East Asia and Pacific; South Asia; Latin America and Caribbean; and United States, Australia and New Zealand. ${ }^{19}$ Countries that did not fit into any of the world regions were categorized as "other." We chose this grouping because we 
hypothesized that differences in donor registration were primarily due to cultural awareness and attitudes.

\section{Statistical analyses}

We compared sociodemographic characteristics and the proportion registered for organ and tissue donation between immigrants and long-term residents using standardized differences, for which a value greater than $10 \%$ indicates a meaningful difference. ${ }^{20}$ We used modified Poisson regression to estimate the prevalence ratio and $95 \%$ confidence intervals (CIs) of organ and tissue donor registration for immigrants relative to longterm residents. ${ }^{21} \mathrm{We}$ also used multivariable modified Poisson regression to identify variables independently associated with organ donor registration among immigrants and long-term residents as well as for the immigrant group only. ${ }^{21} \mathrm{We}$ adjusted for variables chosen a priori (e.g., age, sex, income quintile) based on the findings of a previous study. ${ }^{22} \mathrm{We}$ used the Wilson score method to calculate $95 \%$ CIs for proportions. We conducted complete case analysis (without multiple imputation) for the multivariable analysis because the amount of missing data was low (less than $1.3 \%$ of residents were excluded owing to missing data). We conducted all analyses with SAS software, version 9.3. Finally, we examined the consistency of the associative relations by conducting the analyses stratified by the country of origin of the 5 largest groups of immigrants who had not yet registered for organ and tissue donation.

\section{Ethics approval}

The study was approved by the institutional review board at Sunnybrook Health Sciences Centre, Toronto.

\section{Results}

We identified 1947646 immigrants and 9244570 long-term residents (Appendix 1, available at www.cmajopen.ca/content/4/4/ E551/suppl/DC1). Compared to long-term residents, immigrants were more likely to be younger (mean age $44.1 \mathrm{v}$. 47.3 yr), from lower socioeconomic neighbourhoods and from areas with a higher ethnic concentration; they were less likely to be from rural areas (Table 1). Half of all immigrants were from the East Asia and Pacific region (25.6\%) or South Asia (24.3\%).

\section{Registration for organ and tissue donation}

A total of 231180 immigrants (11.9\%, 95\% CI 11.8-11.9) were registered for deceased organ and tissue donation, compared with 2453116 long-term residents (26.5\%, 95\% CI 26.5-26.6) (Table 2).

When we assessed the proportion of immigrants registered for deceased organ donation according to world region of origin, the region with the highest proportion of registered donors was United States, Australia and New Zealand (40.0\%, 95\% CI 39.5-40.5), followed by Western Europe (20.6\%, 95\% CI 20.4-20.9) and Latin America and Caribbean (15.2\%, 95\% CI 15.1-15.4). Less than $10 \%$ of immigrants from Eastern Europe and Central Asia (9.4\%, 95\% CI 9.29.5), East Asia and Pacific (8.4\%, 95\% CI 8.3-8.5) and subSaharan Africa (7.9\%, 95\% CI 7.7-8.0) were registered.

\section{Characteristics associated with registration}

For the overall group of immigrants and long-term residents combined, those aged 30-39 years had the highest donor registration rate $(29.2 \%$; adjusted prevalence ratio $1.96,95 \%$ CI 1.94-1.99; referent 16-19 yr) (Table 2). In adjusted analyses, we observed no association between income and registration rates. There was a gradient with ethnic concentration and material deprivation: people living in areas with a higher ethnic concentration were less likely to register for organ donation. For example, $14.8 \%$ of people living in the area with the highest ethnic concentration were registered, compared to $29.2 \%$ of those living in the middle quintile (adjusted prevalence ratio $0.58,95 \%$ CI $0.57-0.58$ ) (Table 2). We also found a weak association between 2 of the 4 measures of marginalization (residential instability and dependency) and donor registration (results not shown).

Characteristics among the immigrant population associated with a higher likelihood of registration included economic immigrant status, living in a rural area (population $<10000$ ), living in an area with a lower ethnic concentration, less material deprivation, a higher education, ability to speak English and French, and more years residing in Canada (Table 3). Separated, divorced or widowed immigrants were less likely to register than married immigrants in the unadjusted model but were more likely to register in the adjusted model. In general, immigrants who had been living in Canada for longer periods were more likely to be registered than those living in Canada for less than 4 years.

The 5 countries of birth with the highest absolute numbers of unregistered immigrants were India (202 548 unregistered [13.7\% registered, 95\% CI 13.6-13.9]), China (186 678 [6.4\%, 95\% CI 6.3-6.6]), the Philippines (125 686 [8.5\%, 95\% CI 8.4-8.7]), Pakistan (95 667 [5.8\%, 95\% CI 5.7-6.0]) and Sri Lanka (72 304 [14.7\%, 95\% CI 14.5-15.0]) (Table 4). In our 5 stratified models, we observed effect modification by country of birth for each examined characteristic, which suggests that each characteristic associated somewhat differently with donor registration across these 5 groups. The following characteristics were associated with a higher chance of donor registration in each of the 5 groups: age categories 20-29 years, 30-39 years and 40-49 years (v. 16-19 yr), longer time since arrival in Canada (v. < 4 yr), English and English/French language ability (v. not able to speak either language) and economic immigrant (v. family immigrant). Except for immigrants born in Pakistan, living in the area with the highest ethnic concentration was also associated with lower registration, and higher educational qualifications were associated with higher registration rates.

\section{Exclusion of organs and tissues}

During the donor registration process, when given the option to exclude certain organs and tissues from deceased organ donation, 53473 immigrants (23.1\%) and 409389 long-term residents (16.7\%) excluded at least 1 organ or tissue (Appendix 2, available at www.cmajopen.ca/content/4/4/E551/supp1/DC1). South Asian donors were the most likely to exclude an organ or tissue or both (15 267 people [28.8\%]). Across all groups, the most commonly 


\begin{tabular}{|c|c|c|c|}
\hline \multirow[b]{2}{*}{ Characteristic } & \multicolumn{2}{|c|}{ No. $(\%)$ of people* } & \multirow[b]{2}{*}{$\begin{array}{c}\text { Standardizec } \\
\text { difference, } \\
\% \dagger\end{array}$} \\
\hline & $\begin{array}{c}\text { Immigrants } \\
(n=1947646)\end{array}$ & $\begin{array}{l}\text { Long-term } \\
\text { residents } \\
(n=9244570)\end{array}$ & \\
\hline Age, yr, mean \pm standard deviation & $44.1 \pm 15.5$ & $47.3 \pm 19.2$ & 17 \\
\hline \multicolumn{4}{|l|}{ Age category, yr } \\
\hline $16-19$ & 76073 (3.9) & $599264(6.6)$ & 12 \\
\hline $20-29$ & $290314(14.9)$ & $1510757(16.3)$ & 4 \\
\hline 30-39 & $419949(21.6)$ & $1380229(14.9)$ & 17 \\
\hline $40-49$ & $493544(25.3)$ & $1498785(16.2)$ & 23 \\
\hline $50-59$ & $370569(19.0)$ & $1657095(17.9)$ & 3 \\
\hline $60-69$ & $163899(8.4)$ & $1301436(14.1)$ & 18 \\
\hline $70-79$ & $86024(4.4)$ & $775156(8.4)$ & 16 \\
\hline$\geq 80$ & $47274(2.4)$ & $521848(5.6)$ & 16 \\
\hline Female sex & 933639 (47.9) & 4841077 (52.4) & 9 \\
\hline Rural residenceł & $24848(1.3)$ & $1243904(13.4)$ & 48 \\
\hline \multicolumn{4}{|l|}{ Neighbourhood income quintile } \\
\hline First (lowest) & $493294(25.3)$ & $1618342(17.5)$ & 19 \\
\hline Second & $428901(22.0)$ & $1760076(19.0)$ & 7 \\
\hline Third & $409244(21.0)$ & $1864728(20.2)$ & 2 \\
\hline Fourth & $368900(18.9)$ & 1984856 (21.5) & 6 \\
\hline Fifth (highest) & $247307(12.7)$ & 2016568 (21.8) & 24 \\
\hline \multicolumn{4}{|l|}{ Residential instability quintile } \\
\hline First (lowest) & $604813(31.0)$ & 2350648 (25.4) & 13 \\
\hline Second & $350284(18.0)$ & $1969402(21.3)$ & 8 \\
\hline Third & $207937(10.7)$ & 1542066 (16.7) & 18 \\
\hline Fourth & 335781 (17.2) & 1636146 (17.7) & 1 \\
\hline Fifth (highest) & 433725 (22.3) & 1615948 (17.5) & 12 \\
\hline Missing & $15106(0.8)$ & $130360(1.4)$ & 6 \\
\hline \multicolumn{4}{|l|}{ Ethnic concentration quintile } \\
\hline First (lowest) & $40269(2.1)$ & $1280350(13.8)$ & 45 \\
\hline Second & 73994 (3.8) & 1620959 (17.5) & 46 \\
\hline Third & $125735(6.4)$ & 1749603 (18.9) & 38 \\
\hline Fourth & $294318(15.1)$ & 1963863 (21.2) & 16 \\
\hline Fifth (highest) & $1398224(71.8)$ & 2499435 (27.0) & 100 \\
\hline Missing & $15106(0.8)$ & $130360(1.4)$ & 6 \\
\hline \multicolumn{4}{|l|}{ Dependency quintile } \\
\hline First (lowest) & 663665 (34.1) & $1977392(21.4)$ & 29 \\
\hline Second & $529973(27.2)$ & 2034206 (22.0) & 12 \\
\hline Third & 335353 (17.2) & $1815183(19.6)$ & 6 \\
\hline Fourth & $214055(11.0)$ & 1609352 (17.4) & 18 \\
\hline Fifth (highest) & $189494(9.7)$ & 1678077 (18.2) & 24 \\
\hline Missing & $15106(0.8)$ & $130360(1.4)$ & 6 \\
\hline \multicolumn{4}{|l|}{ Material deprivation quintile } \\
\hline First (lowest) & $502397(25.8)$ & 2461225 (26.6) & 2 \\
\hline Second & $402011(20.6)$ & 2135074 (23.1) & 6 \\
\hline Third & 377933 (19.4) & $1825100(19.7)$ & 1 \\
\hline Fourth & $331916(17.0)$ & 1488359 (16.1) & 2 \\
\hline Fifth (highest) & $318283(16.3)$ & $1204452(13.0)$ & 9 \\
\hline Missing & $15106(0.8)$ & $130360(1.4)$ & 6 \\
\hline
\end{tabular}




\begin{tabular}{|c|c|c|c|}
\hline \multirow[b]{2}{*}{ Characteristic } & \multicolumn{2}{|c|}{ No. (\%) of people* } & \multirow[b]{2}{*}{$\begin{array}{c}\text { Standardized } \\
\text { difference, } \\
\% \dagger\end{array}$} \\
\hline & $\begin{array}{c}\text { Immigrants } \\
(n=1947646)\end{array}$ & $\begin{array}{l}\text { Long-term } \\
\text { residents } \\
(n=9244570)\end{array}$ & \\
\hline \multicolumn{4}{|l|}{ World region of birth } \\
\hline East Asia and Pacific & $499533(25.6)$ & - & - \\
\hline South Asia & $474101(24.3)$ & - & - \\
\hline Latin America and Caribbean & $269170(13.8)$ & - & - \\
\hline Eastern Europe and Central Asia & $215856(11.1)$ & - & - \\
\hline Middle East and North Africa & $181565(9.3)$ & - & - \\
\hline Western Europe & $153259(7.9)$ & - & - \\
\hline Sub-Saharan Africa & $115371(5.9)$ & - & - \\
\hline United States, Australia and New Zealand & $38014(2.0)$ & - & - \\
\hline Other & $777(<0.1)$ & - & - \\
\hline \multicolumn{4}{|l|}{ Education } \\
\hline University degree or higher & $502234(25.8)$ & - & - \\
\hline Some university & $80655(4.1)$ & - & - \\
\hline Nonuniversity qualifications (e.g., college diploma) & $277160(14.2)$ & - & - \\
\hline Secondary or less & $923002(47.4)$ & - & - \\
\hline None & $164521(8.4)$ & - & - \\
\hline Missing & $74(<0.01)$ & - & - \\
\hline \multicolumn{4}{|l|}{ Time since arrival in Canada, yr } \\
\hline$\geq 20$ & $512570(26.3)$ & - & - \\
\hline $15-19$ & $379567(19.5)$ & - & - \\
\hline $10-14$ & $453966(23.3)$ & - & - \\
\hline $4-9$ & $518677(26.6)$ & - & - \\
\hline$<4$ & $82866(4.2)$ & - & - \\
\hline \multicolumn{4}{|l|}{ Language ability } \\
\hline English & $1149609(59.0)$ & - & - \\
\hline French & $16612(0.8)$ & - & - \\
\hline Both & $49192(2.5)$ & - & - \\
\hline Neither & $732166(37.6)$ & - & - \\
\hline Missing & $67(<0.01)$ & - & - \\
\hline \multicolumn{4}{|l|}{ Marital status } \\
\hline Married & 1035265 (53.2) & - & - \\
\hline Separated, divorced, widowed & $74680(3.8)$ & - & - \\
\hline Single & $837396(43.0)$ & - & - \\
\hline Missing & $305(<0.1)$ & - & - \\
\hline \multicolumn{4}{|l|}{ Immigrant class } \\
\hline Economic & $899634(46.2)$ & - & - \\
\hline Family & $685080(35.2)$ & - & - \\
\hline Refugee & $312174(16.0)$ & - & - \\
\hline Other & $50750(2.6)$ & - & - \\
\hline Missing & $8(<0.01)$ & - & - \\
\hline \multicolumn{4}{|c|}{$\begin{array}{l}\text { `Except for age (mean } \pm \text { standard deviation). } \\
\text { †Compared to long-term residents. Standardized differences greater than } 10 \% \text { represent a meaningful difference between } \\
\text { the } 2 \text { groups. } \\
\ddagger \text { Areas with a population less than } 10000 \text {. }\end{array}$} \\
\hline
\end{tabular}




\begin{tabular}{|c|c|c|c|}
\hline \multirow[b]{2}{*}{ Characteristic } & \multirow[b]{2}{*}{ No. (\%) registered } & \multicolumn{2}{|c|}{$\begin{array}{c}\text { Prevalence ratio } \\
\text { (95\% confidence interval) }\end{array}$} \\
\hline & & Unadjusted & Adjusted* \\
\hline \multicolumn{4}{|l|}{ World region of birth } \\
\hline $\begin{array}{l}\text { Long-term residents } \\
(n=9244570)\end{array}$ & $2453116(26.5)$ & $1.00[$ Reference $]$ & 1.00 [Reference] \\
\hline Immigrants ( $n=1947646)$ & $231180(11.9)$ & & \\
\hline East Asia and Pacific & $41752(8.4)$ & $0.31(0.31-0.32)$ & $0.39(0.38-0.40)$ \\
\hline South Asia & $53077(11.2)$ & $0.42(0.42-0.43)$ & $0.53(0.52-0.54)$ \\
\hline Latin America and Caribbean & $41006(15.2)$ & $0.57(0.57-0.58)$ & $0.68(0.67-0.69)$ \\
\hline Eastern Europe and Central Asia & $20222(9.4)$ & $0.35(0.35-0.36)$ & $0.38(0.37-0.40)$ \\
\hline Middle East and North Africa & $19059(10.5)$ & $0.40(0.39-0.40)$ & $0.47(0.46-0.49)$ \\
\hline Western Europe & 31637 (20.6) & $0.78(0.77-0.79)$ & $0.79(0.78-0.81)$ \\
\hline Sub-Saharan Africa & $9080(7.9)$ & $0.30(0.29-0.30)$ & $0.35(0.33-0.36)$ \\
\hline $\begin{array}{l}\text { United States, Australia and New } \\
\text { Zealand }\end{array}$ & $15209(40.0)$ & $1.51(1.49-1.53)$ & $1.40(1.36-1.43)$ \\
\hline Other & $138(17.8)$ & $0.67(0.58-0.78)$ & $1.01(0.74-1.36)$ \\
\hline \multicolumn{4}{|l|}{ Age category, yr } \\
\hline $16-19$ & $107575(15.9)$ & $1.00[$ Reference $]$ & $1.00[$ Reference] \\
\hline $20-29$ & $406873(22.6)$ & $1.42(1.41-1.43)$ & $1.45(1.43-1.47)$ \\
\hline 30-39 & $526486(29.2)$ & $1.84(1.83-1.85)$ & $1.96(1.94-1.99)$ \\
\hline $40-49$ & $556450(27.9)$ & $1.75(1.74-1.76)$ & $1.85(1.82-1.87)$ \\
\hline $50-59$ & $502942(24.8)$ & $1.56(1.55-1.57)$ & $1.54(1.52-1.56)$ \\
\hline $60-69$ & 349575 (23.9) & $1.50(1.49-1.51)$ & $1.41(1.39-1.43)$ \\
\hline $70-79$ & 165279 (19.2) & $1.20(1.20-1.21)$ & $1.11(1.10-1.13)$ \\
\hline$\geq 80$ & $69116(12.1)$ & $0.76(0.76-0.77)$ & $0.69(0.68-0.70)$ \\
\hline \multicolumn{4}{|l|}{ Sex } \\
\hline Female & $1495776(25.9)$ & $1.00[$ Reference] & $1.00[$ Reference] \\
\hline Male & $1188520(21.9)$ & $0.85(0.85-0.85)$ & $0.83(0.83-0.83)$ \\
\hline \multicolumn{4}{|l|}{ Residence } \\
\hline Urban & 2306304 (23.2) & $1.00[$ Reference $]$ & $1.00[$ Reference] \\
\hline Rural† & $377992(29.8)$ & $1.28(1.28-1.29)$ & $0.97(0.96-0.98)$ \\
\hline \multicolumn{4}{|l|}{ Neighbourhood income quintile } \\
\hline First (lowest) & $430400(20.4)$ & $0.86(0.86-0.86)$ & $1.03(1.02-1.04)$ \\
\hline Second & $491648(22.5)$ & $0.95(0.95-0.95)$ & $1.00(0.99-1.01)$ \\
\hline Third & $537122(23.6)$ & $1.00[$ Reference] & $1.00[$ Reference $]$ \\
\hline Fourth & $588534(25.0)$ & $1.06(1.05-1.06)$ & $1.00(0.99-1.00)$ \\
\hline Fifth (highest) & $636592(28.1)$ & $1.19(1.18-1.19)$ & $1.02(1.01-1.02)$ \\
\hline \multicolumn{4}{|l|}{ Ethnic concentration quintile† } \\
\hline First (lowest) & $417114(31.6)$ & $1.08(1.08-1.08)$ & $1.11(1.10-1.11)$ \\
\hline Second & $524728(31.0)$ & $1.06(1.06-1.06)$ & $1.07(1.06-1.07)$ \\
\hline Third & $548379(29.2)$ & $1.00[$ Reference] & $1.00[$ Reference $]$ \\
\hline Fourth & $582938(25.8)$ & $0.88(0.88-0.89)$ & $0.89(0.89-0.89)$ \\
\hline Fifth (highest) & $575086(14.8)$ & $0.50(0.50-0.51)$ & $0.58(0.57-0.58)$ \\
\hline \multicolumn{4}{|l|}{ Material deprivation quintile† } \\
\hline First (lowest) & $772427(26.1)$ & $1.11(1.10-1.11)$ & $1.10(1.10-1.11)$ \\
\hline Second & $625442(24.7)$ & $1.05(1.04-1.05)$ & $1.02(1.01-1.03)$ \\
\hline Third & $519271(23.6)$ & 1.00 [Reference] & 1.00 [Reference] \\
\hline Fourth & 414418 (22.3) & $0.97(0.96-0.97)$ & $1.00(0.99-1.01)$ \\
\hline Fifth (highest) & 316687 (20.8) & $0.88(0.88-0.89)$ & $0.99(0.98-1.01)$ \\
\hline $\begin{array}{l}\text { *Adjusted for world region of birth, sex, } \\
\text { deprivation quintile and ethnic concentr } \\
2238443 \text { ). } \\
\text { †Data missing for } 145466 \text { people ( } 1.3 \%\end{array}$ & $\begin{array}{l}\text { ence, age categor } \\
\text { quintile. Adjusted } \\
\text { whom } 36051 \text { wer }\end{array}$ & $\begin{array}{l}\text { ighbourhood income } \\
\text { sis based on a rand } \\
\text { istered for donor anc }\end{array}$ & $\begin{array}{l}\text { tile, material } \\
\text { ample of } 20 \%(n= \\
\text { ue donation. }\end{array}$ \\
\hline
\end{tabular}


excluded organ or tissue were skin and eyes (Appendix 3, available at www.cmajopen.ca/content/4/4/E551/suppl/DC1). Older people, men and those living in rural areas were less likely to exclude an organ or tissue. Residential instability, dependency and material deprivation showed no clear relation with higher rates of organ or tissue exclusion (results not shown).

\section{Interpretation}

In this cross-sectional study, we documented that immigrants in Ontario had lower rates of registration for organ and tissue donation than did long-term residents of the province. Immigrants born in the United States, Australia and New Zealand

\begin{tabular}{|c|c|c|c|}
\hline \multirow[b]{2}{*}{ Characteristic } & \multirow[b]{2}{*}{ No. (\%) registered* } & \multicolumn{2}{|c|}{ Prevalence ratio ( $95 \%$ confidence interval) } \\
\hline & & Unadjusted & Adjusted $\dagger$ \\
\hline \multicolumn{4}{|l|}{ World region of birth } \\
\hline East Asia and Pacific & $41748(8.4)$ & $0.21(0.21-0.21)$ & $0.28(0.27-0.28)$ \\
\hline South Asia & $53066(11.2)$ & $0.28(0.28-0.28)$ & $0.37(0.36-0.38)$ \\
\hline Latin America and Caribbean & 40985 (15.2) & $0.38(0.38-0.39)$ & $0.51(0.50-0.52)$ \\
\hline Eastern Europe and Central Asia & $20216(9.4)$ & $0.23(0.23-0.24)$ & $0.28(0.27-0.28)$ \\
\hline Middle East and North Africa & $19056(10.5)$ & $0.26(0.26-0.27)$ & $0.33(0.32-0.33)$ \\
\hline Western Europe & $31634(20.6)$ & $0.52(0.51-0.52)$ & $0.57(0.56-0.58)$ \\
\hline Sub-Saharan Africa & $9078(7.9)$ & $0.20(0.19-0.20)$ & $0.26(0.26-0.27)$ \\
\hline $\begin{array}{l}\text { United States, Australia and } \\
\text { New Zealand }\end{array}$ & $15207(40.0)$ & $1.00[$ Reference $]$ & $1.00[$ Reference] \\
\hline Other & $138(17.8)$ & $0.44(0.38-0.52)$ & $0.58(0.50-0.67)$ \\
\hline \multicolumn{4}{|l|}{ Age category, yr } \\
\hline $16-19$ & $4545(6.0)$ & 1.00 [Reference] & 1.00 [Reference] \\
\hline $20-29$ & $31791(11.0)$ & $1.83(1.78-1.89)$ & $1.85(1.80-1.91)$ \\
\hline 30-39 & $57841(13.8)$ & $2.31(2.24-2.37)$ & $2.23(2.16-2.30)$ \\
\hline $40-49$ & $68319(13.8)$ & $2.32(2.25-2.39)$ & $2.02(1.95-2.08)$ \\
\hline $50-59$ & $44244(11.9)$ & $2.00(1.94-2.06)$ & $1.76(1.70-1.82)$ \\
\hline $60-69$ & $16302(9.9)$ & $1.66(1.61-1.72)$ & $1.57(1.52-1.63)$ \\
\hline $70-79$ & $5931(6.9)$ & $1.15(1.11-1.20)$ & $1.28(1.23-1.33)$ \\
\hline$\geq 80$ & $2155(4.6)$ & $0.76(0.73-0.80)$ & $0.88(0.84-0.93)$ \\
\hline \multicolumn{4}{|l|}{ Sex } \\
\hline Female & $121402(12.0)$ & 1.00 [Reference] & 1.00 [Reference] \\
\hline Male & $109726(11.8)$ & $0.98(0.97-0.99)$ & $0.93(0.93-0.94)$ \\
\hline \multicolumn{4}{|l|}{ Residence } \\
\hline Urban & $224266(11.7)$ & 1.00 [Reference] & 1.00 [Reference] \\
\hline Rural & $6862(28.3)$ & $2.43(2.38-2.48)$ & $1.24(1.21-1.26)$ \\
\hline \multicolumn{4}{|l|}{ Neighbourhood income quintile } \\
\hline First (lowest) & $43634(8.8)$ & $0.73(0.72-0.74)$ & $0.96(0.94-0.97)$ \\
\hline Second & $46543(10.9)$ & $0.90(0.89-0.91)$ & $1.00(0.99-1.01)$ \\
\hline Third & $49520(12.1)$ & 1.00 [Reference] & 1.00 [Reference] \\
\hline Fourth & $49308(13.4)$ & $1.10(1.09-1.12)$ & $1.09(1.08-1.11)$ \\
\hline Fifth (highest) & $42123(17.0)$ & $1.41(1.39-1.42)$ & $1.02(1.00-1.03)$ \\
\hline \multicolumn{4}{|l|}{ Ethnic concentration quintileł } \\
\hline First (lowest) & 9435 (23.4) & $1.27(1.25-1.30)$ & $1.12(1.10-1.15)$ \\
\hline Second & $15272(20.6)$ & $1.12(1.10-1.14)$ & $1.06(1.04-1.08)$ \\
\hline Third & 23119 (18.4) & 1.00 [Reference] & 1.00 [Reference] \\
\hline Fourth & 43565 (14.8) & $0.81(0.79-0.82)$ & $0.88(0.86-0.89)$ \\
\hline Fifth (highest) & $137715(9.9)$ & $0.54(0.53-0.54)$ & $0.70(0.69-0.71)$ \\
\hline
\end{tabular}




\begin{tabular}{|c|c|c|c|}
\hline \multirow[b]{2}{*}{ Characteristic } & \multirow[b]{2}{*}{ No. (\%) registered ${ }^{\star}$} & \multicolumn{2}{|c|}{ Prevalence ratio ( $95 \%$ confidence interval) } \\
\hline & & Unadjusted & Adjusted $†$ \\
\hline \multicolumn{4}{|l|}{ Material deprivation quintileł } \\
\hline First (lowest) & $72247(14.4)$ & $1.23(1.21-1.24)$ & $1.09(1.08-1.10)$ \\
\hline Second & $524 a 77(13.1)$ & $1.11(1.10-1.13)$ & $1.04(1.03-1.05)$ \\
\hline Third & 44268 (11.9) & 1.00 [Reference] & 1.00 [Reference] \\
\hline Fourth & $33461(10.1)$ & $0.86(0.85-0.87)$ & $0.92(0.91-0.93)$ \\
\hline Fifth (highest) & $26653(8.4)$ & $0.71(0.70-0.73)$ & $0.82(0.80-0.83)$ \\
\hline \multicolumn{4}{|l|}{ Education§ } \\
\hline University degree or higher & 71901 (14.3) & 1.00 [Reference] & 1.00 [Reference] \\
\hline Some university & $11142(13.8)$ & $0.97(0.95-0.98)$ & $0.96(0.95-0.98)$ \\
\hline $\begin{array}{l}\text { Nonuniversity qualifications } \\
\text { (e.g., college diploma) }\end{array}$ & $36403(13.1)$ & $0.92(0.91-0.93)$ & $0.92(0.91-0.93)$ \\
\hline Secondary or less & $95818(10.4)$ & $0.73(0.72-0.73)$ & $0.78(0.77-0.79)$ \\
\hline None & $15864(9.6)$ & $0.67(0.66-0.68)$ & $0.81(0.80-0.83)$ \\
\hline \multicolumn{4}{|l|}{ Time since arrival in Canada, yr } \\
\hline$\geq 20$ & $56371(11.0)$ & $1.23(1.21-1.26)$ & $1.31(1.28-1.35)$ \\
\hline $15-19$ & $53046(14.0)$ & $1.57(1.53-1.61)$ & $1.76(1.72-1.80)$ \\
\hline $10-14$ & $60633(13.4)$ & $1.50(1.47-1.53)$ & $1.66(1.62-1.70)$ \\
\hline $4-9$ & $53695(10.4)$ & $1.16(1.14-1.19)$ & $1.21(1.18-1.23)$ \\
\hline$<4$ & $7383(8.9)$ & $1.00[$ Reference] & $1.00[$ Reference] \\
\hline \multicolumn{4}{|l|}{ Language ability§ } \\
\hline English & $160835(14.0)$ & $1.00[$ Reference $]$ & $1.00[$ Reference] \\
\hline French & $1305(7.9)$ & $0.56(0.53-0.59)$ & $0.66(0.63-0.70)$ \\
\hline Both & $7704(15.7)$ & $1.12(1.10-1.14)$ & $1.06(1.04-1.08)$ \\
\hline Neither & $61284(8.4)$ & $0.60(0.59-0.60)$ & $0.76(0.75-0.77)$ \\
\hline \multicolumn{4}{|l|}{ Marital status§ } \\
\hline Married & $121619(11.7)$ & $1.00[$ Reference] & 1.00 [Reference] \\
\hline Separated, divorced, widowed & $6876(9.2)$ & $0.78(0.77-0.80)$ & $1.06(1.04-1.09)$ \\
\hline Single & $102633(12.3)$ & $1.04(1.04-1.05)$ & $1.11(1.10-1.12)$ \\
\hline \multicolumn{4}{|l|}{ Immigrant class§ } \\
\hline Economic & 119029 (13.2) & $1.00[$ Reference] & $1.00[$ Reference] \\
\hline Family & $74731(10.9)$ & $0.82(0.82-0.83)$ & $0.86(0.86-0.87)$ \\
\hline Refugee & $31305(10.0)$ & $0.76(0.75-0.77)$ & $0.95(0.94-0.96)$ \\
\hline Other & $6063(11.9)$ & $0.90(0.88-0.93)$ & $0.96(0.93-0.98)$ \\
\hline \multicolumn{4}{|c|}{$\begin{array}{l}\text { *Total number of immigrants for this analysis was } 1947192 \text {, of whom } 231128 \text { were registered for organ and tissue donation; } \\
454 \text { immigrants, of whom } 52 \text { were registered, had missing data on immigration-related characteristics and were excluded from } \\
\text { this cohort. } \\
\text { †Adjusted for world region of birth, sex, residence, age category, neighbourhood income quintile, material deprivation quintile, } \\
\text { ethnic concentration quintile, education, time since arrival in Canada, language ability, marital status and immigrant class. } \\
\text { łData missing for } 15102 \text { people }(<1.0 \%) \text {, of whom } 2022 \text { were registered. } \\
\text { §Data missing for } 454 \text { people }(<0.01 \%) \text {, of whom } 52 \text { were registered, who were then further excluded from analysis. }\end{array}$} \\
\hline
\end{tabular}

region had the highest registration rates, even higher than those for long-term residents. In addition, among immigrants, age 30-39 years, higher education, ability to speak English or both English and French, economic immigrant status, married or single status, and living in an area with a lower ethnic concentration were associated with higher registration rates. These findings highlight the marked differences in donor registration rates across immigrant groups and inform the development and implementation of targeted, culture-sensitive public campaigns to raise awareness about organ and tissue donation.

Our findings are consistent with those of López and colleagues, ${ }^{23}$ who investigated the attitudes of immigrants in Spain toward deceased organ donation. Like those authors, we found that, among the immigrant population as a whole, women, people with higher education and those with a higher income were more likely to register for organ donation. However, López and 
colleagues $^{23}$ found that immigrants from East Europe and North Africa were more reluctant to donate their organs compared to other immigrant groups, whereas in our study, immigrants born in sub-Saharan Africa and the East Asia and Pacific region were the least likely groups to be registered for organ donation.

Our results are also similar to those of a previous study, in which we used a surname algorithm to identify people of Chinese and South Asian origin in Ontario. ${ }^{24}$

In the current study, many immigrant groups were much less likely to register for organ donation compared with longterm residents, but these differences decreased by up to $10 \%$ in some cases after we adjusted for residential ethnic concentra- tion. Furthermore, living in a rural community, higher neighbourhood income quintile and lower material deprivation quintile were no longer strongly positively associated with donor registration after adjustment for ethnic concentration among all Ontario residents. In another study examining how community-level factors affect rates of registration for organ donation, Ladin and colleagues ${ }^{25}$ found that groups with higher levels of racial homogeneity, native-born residents and other social capital variables had higher registration rates. They suggested that minority populations may have higher rates of altruistic behaviour (i.e., organ donor registration) if they feel less isolated and better integrated with their community.

\begin{tabular}{|c|c|c|c|c|c|}
\hline \multirow[b]{2}{*}{ Characteristic } & \multicolumn{5}{|c|}{ Adjusted prevalence ratio ( $95 \%$ confidence interval) ${ }^{*}$} \\
\hline & India & China & Philippines & Pakistan & Sri Lanka \\
\hline \multicolumn{6}{|l|}{ Age category, yr } \\
\hline $16-19$ & $1.00[$ Reference] & 1.00 [Reference] & $1.00[$ Reference] & 1.00 [Reference] & 1.00 [Reference] \\
\hline $20-29$ & $1.57(1.44-1.71)$ & $1.64(1.43-1.88)$ & $1.74(1.55-1.97)$ & $2.1(1.78-2.48)$ & $1.87(1.61-2.18)$ \\
\hline $30-39$ & $1.69(1.55-1.85)$ & $1.41(1.21-1.63)$ & $1.85(1.63-2.10)$ & $2.1(1.76-2.51)$ & $2.09(1.79-2.44)$ \\
\hline $40-49$ & $1.85(1.69-2.02)$ & $1.28(1.10-1.48)$ & $1.44(1.26-1.65)$ & $2.18(1.82-2.61)$ & $1.75(1.49-2.04)$ \\
\hline $50-59$ & $1.71(1.56-1.87)$ & $1.17(1.01-1.37)$ & $1.10(0.96-1.27)$ & $2.37(1.96-2.87)$ & $1.44(1.23-1.7)$ \\
\hline $60-69$ & $1.40(1.27-1.54)$ & $1.08(0.92-1.28)$ & $0.83(0.71-0.97)$ & $2.56(2.08-3.14)$ & $1.26(1.06-1.49)$ \\
\hline $70-79$ & 1.19 (1.07-1.32) & $0.85(0.71-1.01)$ & $0.54(0.44-0.66)$ & $2.37(1.83-3.09)$ & $1.3(1.08-1.56)$ \\
\hline$\geq 80$ & $0.94(0.82-1.08)$ & $0.58(0.47-0.71)$ & $0.38(0.29-0.49)$ & 2.3 (1.59-3.33) & $0.94(0.75-1.17)$ \\
\hline \multicolumn{6}{|l|}{ Sex } \\
\hline Female & 1.00 [Reference] & 1.00 [Reference] & 1.00 [Reference] & 1.00 [Reference] & 1.00 [Reference] \\
\hline Male & $1.01(0.99-1.03)$ & $1.03(0.99-1.06)$ & $0.81(0.78-0.84)$ & 1.09 (1.03-1.15) & $1.04(1.00-1.08)$ \\
\hline \multicolumn{6}{|l|}{ Residence } \\
\hline Urban & 1.00 [Reference] & 1.00 [Reference] & 1.00 [Reference] & 1.00 [Reference] & 1.00 [Reference] \\
\hline Rural† & $1.09(0.96-1.23)$ & $1.22(0.98-1.52)$ & $1.38(1.16-1.65)$ & $1.41(1.02-1.93)$ & $1.11(0.83-1.48)$ \\
\hline \multicolumn{6}{|c|}{ Neighbourhood income quintile } \\
\hline First (lowest) & $0.90(0.87-0.94)$ & $1.1(1.04-1.18)$ & $0.90(0.85-0.97)$ & $0.89(0.80-0.98)$ & $0.99(0.93-1.06)$ \\
\hline Second & $0.95(0.92-0.98)$ & $1.01(0.95-1.06)$ & $0.96(0.91-1.01)$ & $1.04(0.96-1.13)$ & $0.96(0.92-1.01)$ \\
\hline Third & 1.00 [Reference] & 1.00 [Reference] & 1.00 [Reference] & 1.00 [Reference] & 1.00 [Reference] \\
\hline Fourth & 1.04 (1.01-1.07) & $1.04(0.99-1.1)$ & $1.05(0.99-1.11)$ & $0.96(0.89-1.04)$ & $1.00(0.95-1.06)$ \\
\hline Fifth (highest) & $1.14(1.10-1.18)$ & $1.06(1.00-1.12)$ & $0.98(0.91-1.05)$ & $1.18(1.08-1.30)$ & $1.15(1.07-1.23)$ \\
\hline \multicolumn{6}{|c|}{ Ethnic concentration quintile } \\
\hline First (lowest) & $1.02(0.92-1.12)$ & $1.21(1.04-1.41)$ & $1.08(0.93-1.24)$ & $1.16(0.91-1.49)$ & $1.07(0.87-1.32)$ \\
\hline Second & $1.01(0.94-1.09)$ & $1.16(1.03-1.32)$ & $1.09(0.98-1.22)$ & $0.92(0.76-1.12)$ & $1.07(0.93-1.24)$ \\
\hline Third & 1.00 [Reference] & 1.00 [Reference] & 1.00 [Reference] & 1.00 [Reference] & 1.00 [Reference] \\
\hline Fourth & $0.91(0.87-0.96)$ & $0.96(0.88-1.05)$ & $0.87(0.81-0.95)$ & $1.07(0.94-1.23)$ & $0.89(0.8-0.99)$ \\
\hline Fifth (highest) & $0.82(0.78-0.86)$ & $0.76(0.70-0.82)$ & $0.77(0.72-0.83)$ & $0.88(0.77-0.99)$ & $0.81(0.74-0.89)$ \\
\hline \multicolumn{6}{|c|}{ Material deprivation quintile } \\
\hline First (lowest) & 1.06 (1.02-1.09) & $0.99(0.94-1.05)$ & $1.11(1.05-1.18)$ & $0.97(0.89-1.05)$ & $1.13(1.06-1.19)$ \\
\hline Second & $1.00(0.97-1.03)$ & $1.01(0.96-1.07)$ & $1.01(0.95-1.07)$ & $0.98(0.91-1.06)$ & $1.03(0.98-1.08)$ \\
\hline Third & 1.00 [Reference] & 1.00 [Reference] & 1.00 [Reference] & 1.00 [Reference] & 1.00 [Reference] \\
\hline Fourth & $0.89(0.86-0.92)$ & $0.89(0.84-0.94)$ & $0.93(0.87-0.98)$ & $0.77(0.70-0.85)$ & $0.94(0.89-0.99)$ \\
\hline Fifth (highest) & $0.77(0.74-0.81)$ & $0.86(0.80-0.93)$ & $0.93(0.86-0.99)$ & $0.76(0.68-0.86)$ & $0.86(0.80-0.92)$ \\
\hline
\end{tabular}




\section{OPEN}

Research

\begin{tabular}{|c|c|c|c|c|c|}
\hline \multirow[b]{2}{*}{ Characteristic } & \multicolumn{5}{|c|}{ Adjusted prevalence ratio $\left(95 \%\right.$ confidence interval) ${ }^{\star}$} \\
\hline & India & China & Philippines & Pakistan & Sri Lanka \\
\hline \multicolumn{6}{|l|}{ Education } \\
\hline University degree or higher & $1.00[$ Reference $]$ & 1.00 [Reference] & $1.00[$ Reference $]$ & $1.00[$ Reference $]$ & $1.00[$ Reference $]$ \\
\hline Some university & $0.91(0.86-0.96)$ & $0.87(0.8-0.93)$ & $0.84(0.79-0.91)$ & $1.28(1.11-1.48)$ & $0.88(0.79-0.98)$ \\
\hline $\begin{array}{l}\text { Nonuniversity qualifications } \\
\text { (e.g., college diploma) }\end{array}$ & $0.81(0.78-0.83)$ & $0.55(0.52-0.58)$ & $0.82(0.77-0.86)$ & $0.90(0.84-0.97)$ & $0.70(0.66-0.74)$ \\
\hline Secondary or less & $0.96(0.93-0.99)$ & $0.75(0.71-0.79)$ & $0.78(0.74-0.82)$ & $0.91(0.84-1.00)$ & $0.89(0.83-0.95)$ \\
\hline None & $0.71(0.67-0.75)$ & $0.58(0.52-0.65)$ & $0.76(0.69-0.84)$ & $0.92(0.81-1.05)$ & $0.72(0.66-0.79)$ \\
\hline \multicolumn{6}{|l|}{ Time since arrival in Canada, yr } \\
\hline$\geq 20$ & $1.41(1.32-1.51)$ & $1.78(1.54-2.06)$ & $1.77(1.61-1.95)$ & $2.04(1.63-2.55)$ & $1.48(1.32-1.67)$ \\
\hline $15-19$ & $2.09(1.96-2.23)$ & $3.57(3.14-4.06)$ & $2.35(2.15-2.57)$ & $2.52(2.05-3.09)$ & $1.83(1.64-2.06)$ \\
\hline $10-14$ & $1.97(1.85-2.09)$ & $2.92(2.58-3.31)$ & $2.17(1.99-2.37)$ & $2.04(1.67-2.50)$ & $1.87(1.67-2.10)$ \\
\hline $4-9$ & $1.22(1.15-1.30)$ & $1.37(1.21-1.55)$ & $1.51(1.39-1.65)$ & $1.23(1.01-1.51)$ & $1.19(1.06-1.33)$ \\
\hline$<4$ & $1.00[$ Reference $]$ & 1.00 [Reference] & $1.00[$ Reference $]$ & $1.00[$ Reference $]$ & $1.00[$ Reference $]$ \\
\hline \multicolumn{6}{|l|}{ Language ability } \\
\hline English & $1.00[$ Reference $]$ & $1.00[$ Reference] & $1.00[$ Reference $]$ & $1.00[$ Reference $]$ & $1.00[$ Reference $]$ \\
\hline French & $0.71(0.69-0.73)$ & $0.73(0.70-0.75)$ & $0.93(0.88-0.98)$ & $0.60(0.56-0.65)$ & $0.77(0.74-0.81)$ \\
\hline Both & $1.16(1.04-1.29)$ & $0.93(0.75-1.17)$ & $1.60(1.12-2.28)$ & $1.18(0.86-1.63)$ & $1.02(0.78-1.34)$ \\
\hline Neither & $0.71(0.69-0.73)$ & $0.73(0.70-0.75)$ & $0.93(0.88-0.98)$ & $0.60(0.56-0.65)$ & $0.77(0.74-0.81)$ \\
\hline \multicolumn{6}{|l|}{ Marital status } \\
\hline Married & 1.00 [Reference] & 1.00 [Reference] & 1.00 [Reference] & 1.00 [Reference] & 1.00 [Reference] \\
\hline $\begin{array}{l}\text { Separated, divorced, } \\
\text { widowed }\end{array}$ & $1.09(1.01-1.18)$ & $1.23(1.11-1.37)$ & $1.08(0.96-1.21)$ & $1.10(0.90-1.34)$ & $0.98(0.88-1.10)$ \\
\hline Single & $1.12(1.09-1.15)$ & $1.19(1.14-1.25)$ & $0.97(0.93-1.01)$ & $1.24(1.16-1.33)$ & $1.05(1.01-1.10)$ \\
\hline \multicolumn{6}{|l|}{ Immigrant class } \\
\hline Economic & 1.00 [Reference] & 1.00 [Reference] & 1.00 [Reference] & 1.00 [Reference] & 1.00 [Reference] \\
\hline Family & $0.80(0.78-0.82)$ & $0.86(0.82-0.91)$ & $0.84(0.81-0.88)$ & $0.82(0.76-0.88)$ & $0.73(0.69-0.77)$ \\
\hline Refugee & $0.96(0.89-1.04)$ & $0.73(0.67-0.80)$ & $1.33(0.98-1.79)$ & $1.39(1.28-1.51)$ & $0.83(0.79-0.87)$ \\
\hline Other & $1.04(0.93-1.17)$ & $0.68(0.60-0.76)$ & $1.06(0.90-1.25)$ & $1.33(1.10-1.62)$ & $0.95(0.86-1.05)$ \\
\hline
\end{tabular}

\section{Strengths and limitations}

Our study has several strengths. First, we examined actual rates of registration for organ and tissue donation rather than attitudes toward donation among immigrants as well as characteristics associated with registration. In addition, we accounted for important variables such as age and socioeconomic status when comparing ethnic groups. ${ }^{4}$ Furthermore, most previous studies focused on specific ethnic or cultural collectives such as Hispanics, African Americans, Asians and Arabs, whereas our study focused on the entire immigrant population of a large jurisdiction.

However, our study has important limitations. First, we had no information on the reasons why many immigrants did not register for organ donation, which is important for the design of educational programs. Low registration rates in specific immigrant groups may be influenced by many factors, including knowledge, attitude and awareness of organ donation, that were not measured in our study. The low registration rates may also have been due to unawareness of the registry ${ }^{7,8}$ or fear of placing one's name in a large database $e^{26}$ rather than a negative attitude toward organ donation. Second, Immigration, Refugees and Citizenship Canada's Permanent Resident Database contains only data recorded at the time of immigration. More than $50 \%$ of our cohort arrived in Canada more than 10 years ago, and certain variables such as education, language ability and marital status may have changed over the years. For example, Okrainec and colleagues ${ }^{16}$ found that self-reported language barriers in the Permanent Resident Database are a poor indicator of persistent language barrier compared with the Canadian Community Health Survey. Despite the limitations in our data, strong differences in registration rates between immigrants and long-term residents persisted even after we controlled for many sociodemographic factors. 


\section{Conclusion}

This study documents that fewer Ontario immigrants than long-term residents of the province registered for deceased organ and tissue donation. There is a need to better understand the causes of lower registration rates among various immigrant groups. However, to fill the Ontario donor registry, it is also important to better understand the reasons for the low rate of donor registration among long-term residents, given that they account for a large absolute number of unregistered residents. More research on other community-level factors associated with higher donor registration rates, such as volunteerism and civic participation, is needed. More research is also needed to develop and evaluate culture-tailored interventions that can build support for deceased organ and tissue donation.

\section{References}

1. Marck CH, Neate SL, Skinner MR, et al. Factors relating to consent for organ donation: prospective data on potential organ donors. Intern Med 7 2015;45:40-7.

2. Rosenblum AM, Li AH, Roels L, et al. Worldwide variability in deceased organ donation registries. Transpl Int 2012;25:801-11.

3. Rosenblum AM, Horvat LD, Siminoff LA, et al. The authority of next-of-kin in explicit and presumed consent systems for deceased organ donation: an analysis of 54 nations. Nephrol Dial Transplant 2012;27:2533-46.

4. Morgan M, Kenten C, Deedat S; Donate Programme Team. Attitudes to deceased organ donation and registration as a donor among minority ethnic groups in North America and the U.K.: a synthesis of quantitative and qualitative research. Ethn Health 2013;18:367-90.

5. Boulware LE, Ratner LE, Sosa JA, et al. Determinants of willingness to donate living related and cadaveric organs: identifying opportunities for intervention. Transplantation 2002;73:1683-91.

6. Siminoff LA, Burant CJ, Ibrahim SA. Racial disparities in preferences and perceptions regarding organ donation. 7 Gen Intern Med 2006;21:995-1000.

7. Molzahn AE, Starzomski R, McDonald M, et al. Indo-Canadian beliefs regarding organ donation. Prog Transplant 2005;15:233-9.

8. Molzahn AE, Starzomski R, McDonald M, et al. Chinese Canadian beliefs toward organ donation. Qual Health Res 2005;15:82-98.

9. Padela AI, Rasheed S, Warren GJW, et al. Factors associated with positive attitudes toward organ donation in Arab Americans. Clin Transplant 2011;25:800-8.

10. Immigration and ethnocultural diversity in Canada. Ottawa: Statistics Canada; 2013. Available: https://www12.statcan.gc.ca/nhs-enm/2011/as-sa/99-010 -x/99-010-x2011001-eng.cfm (accessed 2016 May 9).

11. Da Silva IR, Frontera JA. Worldwide barriers to organ donation. FAMA Neurol 2015;72:112-8

12. Demographic quarterly: highlights of first quarter 2016. Oshawa (ON): Ontario Ministry of Finance; 2016. Available: www.fin.gov.on.ca/en/economy/ demographics/quarterly/dhiq1.html (accessed 2016 Aug. 17).

13. Krieger N. Overcoming the absence of socioeconomic data in medical records: validation and application of a census-based methodology. Am 7 Public Health 1992;82:703-10.

14. Ontario Marginalization Index (ON-Marg) - ON-Marg FAQs. Toronto: Toronto Community Health Profiles Partnership. Available: www.torontohealthprofiles.ca/onmarg_faq.php (accessed 2015 Aug. 5).

15. Matheson FI, Dunn JR, Smith KLW, et al. Development of the Canadian Marginalization Index: a new tool for the study of inequality. Can $\mathcal{F}$ Public Health 2012;103:S12-6.

16. Okrainec K, Booth GL, Hollands S, et al. Language barriers among the foreign-born in Canada: agreement of self-reported measures and persistence over time. 7 Immigr Minor Health 2015 [Epub ahead of print]. DOI: 10.1007/ s10903-015-0279-9.

17. Creatore MI, Moineddin R, Booth G, et al. Age- and sex-related prevalence of diabetes mellitus among immigrants to Ontario, Canada. CMAJ 2010;182:781-9.

18. Lofters AK, Moineddin R, Hwang SW, et al. Predictors of low cervical cancer screening among immigrant women in Ontario, Canada. BMC Womens Health 2011;11:20.

19. Countries. Washington: The World Bank; 2015. Available: www.worldbank. org/en/country (accessed 2015 June 30).

20. Austin PC. Using the standardized difference to compare the prevalence of a binary variable between two groups in observational research. Commun Stat Simul Comput 2009;38:1228-34.

21. Zou G. A modified Poisson regression approach to prospective studies with binary data. Am 7 Epidemiol 2004;159:702-6.

22. Wakefield CE, Watts KJ, Homewood J, et al. Attitudes toward organ donation and donor behavior: a review of the international literature. Prog Transplant 2010;20:380-91.
23. López JS, Valentín MO, Scandroglio B, et al. Factors related to attitudes toward organ donation after death in the immigrant population in Spain. Clin Transplant 2012;26:E200-12.

24. Li AH, McArthur E, Maclean J, et al. Deceased organ donation registration and familial consent among Chinese and South Asians in Ontario, Canada. PLoS One 2015;10:e124321.

25. Ladin K, Wang R, Fleishman A, et al. Does social capital explain communitylevel differences in organ donor designation? Milbank Q 2015;93:609-41.

26. Siegel JT, Alvaro EM, Jones SP. Organ donor registration preferences among Hispanic populations: Which modes of registration have the greatest promise? Health Educ Behav 2005;32:242-52.

Affiliations: Department of Epidemiology and Biostatistics (Li, Weir, Garg), Western University, London, Ont.; Division of Nephrology (Lam), University of Alberta, Edmonton, Alta.; Trillium Gift of Life Network (Dhanani, Prakash), Toronto, Ont.; Division of Critical Care (Dhanani), Children's Hospital of Eastern Ontario, University of Ottawa, Ottawa, Ont.; Division of Nephrology (Weir, Garg), Western University, London, Ont.; Division of Nephrology (Kim), Department of Medicine, University of Toronto, Toronto, Ont.; Division of Nephrology and Ottawa Hospital Research Institute (Knoll), The Ottawa Hospital and University of Ottawa, Ottawa, Ont.; Institute for Clinical Evaluative Sciences (Garg), London, Ont.

Contributors: Alvin Li and Amit Garg contributed substantially to the conception and design of the study, acquired the data and drafted the manuscript. Alvin Li analyzed the data. All of the authors contributed to data interpretation. Ngan Lam, Sonny Dhanani, Matthew Weir, Versha Prakash, Joseph Kim and Greg Knoll revised the manuscript critically for important intellectual content. All of the authors approved the final version to be published and agreed to act as guarantors of the work.

Funding: Amit Garg received an investigator-initiated grant from Astellas Pharma Canada, Inc. and Roche Canada to support a Canadian Institutes of Health Research study in living kidney donors, and his institution received unrestricted research funding from Pfizer Canada. He is the Dr. Adam Linton Chair in Kidney Health Analytics, Schulich School of Medicine and Dentistry and was supported through an award from the Academic Medical Organization of Southwestern Ontario. Alvin Li is a trainee of the Canadian National Transplant Research Program and was supported by a doctoral scholarship from the Kidney Foundation of Canada and the Canadian Institutes of Health Research. Ngan Lam was supported by a KRESCENT New Investigator Award. Greg Knoll is a Research Chair in Clinical Transplantation Research at the University of Ottawa.

Acknowledgements: This Institute for Clinical Evaluative Sciences (ICES) project was conducted by the Kidney, Dialysis and Transplantation program at the ICES Western site. Parts of this material were based on data and information compiled and provided by the Canadian Institute for Health Information; however, the analyses, conclusions, opinions and statements expressed herein are those of the authors and not necessarily those of the Canadian Institute for Health Information. The authors thank Immigration, Refugees and Citizenship Canada for providing access to the databases used in this study. Core funding for ICES Western is provided by the Academic Medical Organization of Southwestern Ontario, the Schulich School of Medicine and Dentistry, Western University and the Lawson Health Research Institute. The ICES Kidney, Dialysis and Transplantation receives program operating grant support from the Canadian Institutes of Health Research. Aspects of this project were conducted in the Lilibeth Caberto Kidney Clinical Research Unit. The opinions, results and conclusions are those of the authors and are independent from the funding sources. No endorsement by these organizations is intended or should be inferred. The funders had no role in study design, data collection and analysis, decision to publish or preparation of the manuscript.

ICES disclaimer: This study was supported by the Institute for Clinical Evaluative Sciences (ICES), which is funded by an annual grant from the Ontario Ministry of Health and Long-Term Care (MOHLTC). The opinions, results and conclusions reported in this article are those of the authors and are independent from the funding sources. No endorsement by the ICES or the Ontario MOHLTC is intended or should be inferred.

Supplemental information: For reviewer comments and the original submission of this manuscript, please see www.cmajopen.ca/content/4/4/ E551/suppl/DC1 